\title{
Diagnostic Test of Large-Scale SFQ Shift Register
}

\author{
Hirotaka Terai, Masamitsu Tanaka, Yuuki Yamanashi, Yoshihito Hashimoto, Akira Fujimaki, \\ Nobuyuki Yoshikawa, and Zhen Wang
}

\begin{abstract}
We diagnosed circuits to identify of margin reduction mechanisms in large-scale SFQ circuits. Two types of shift registers with partial or full bias line shields were designed and tested. We investigated the locations and origins of circuit errors by measuring bias margins and output waveforms. The results indicated that bias line currents can degrade the bias margin around the periphery in large-scale circuits with partial bias line shields. On the other hand, no apparent reduction of bias margins was observed in any part of the circuit area in the circuits with full bias line shields. However, we observed a reduction of the bias margin due to flux trapping even when we used a completely non-magnetic He dewar surrounded by a dual-layer permalloy metal shield. We found that the probability of finding a defect in a circuit with 10,000 Josephson junctions is less than $60 \%$.
\end{abstract}

Index Terms-Bias current supply, circuit diagnostics, fabrication yield, flux trapping, single-flux-quantum circuit.

\section{INTRODUCTION}

$\mathbf{R}$ ECENT advances in single-flux-quantum (SFQ) circuit technology have made integrated circuits including 10,000 Josephson junctions (JJs), possible [1], [2]. In principle, over one million Josephson junctions may be integrated on a 10 -mm square chip by reducing feature size and increasing the number of superconductor layers [3], [4]. However, various factors, such as magnetic fields arising from bias currents, flux trapping, and fabrication yield, can degrade overall operating margins [5]-[9]. Depending on the type of circuit, the operating margin of a circuit consisting of about $10,000 \mathrm{JJs}$ is usually narrow, typically less than $+/-10 \%$, while those for the individual logic gate are more than $+/-25 \%$.

One of the problems in testing large-scale SFQ circuits is identifying the origins and locations of circuit errors. An error often spreads to other parts of the circuit and degrades its operation, making testing difficult. Direct probes can reveal what happens in the circuits, but it is hard to use direct probes in the cryogenic environments required for circuit operations. In addition, since signals in SFQ circuits are tiny voltage pulses, a circuit to convert such pulses into static voltage is necessary to monitor them. Furthermore, to prevent thermal inflow, the number

Manuscript received August 29, 2006. This work was supported by the Ministry of Economy, Trade and Industry through ISTEC as part of the Superconductor Network Device Project.

H. Terai and Z. Wang are with the Kobe Advanced Research Center, National Institute of Information and Communications technology, Kobe 651-2492, Japan (e-mail: terai@nict.go.jp).

M. Tanaka and A. Fujimaki are with the Department of Quantum Engineering, Nagoya University, Furo-cho, Chikusaku, Nagoya 464-8603, Japan.

Y. Yamanashi and N. Yoshikawa is with the Department of Computer Engineering, Yokohama National University, Tokiwadai, Hodogayaku, Yokohama 464-8603, Japan.

Y. Hashimoto is with the Superconducting Research Lab., International Superconductivity Research Center, 34 Miyukigaoka, Tsukuba, Ibaraki 305-8501, Japan.

Digital Object Identifier 10.1109/TASC.2007.898559

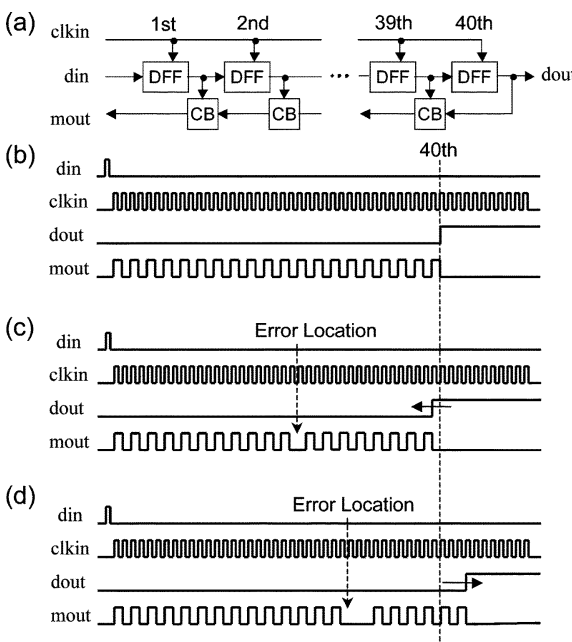

Fig. 1. (a) Block diagram of diagnostic shift register and expected operations for (b) optimal bias, (c) over bias, and (d) under bias.

of signal lines from the cryogenic environment to the measurement system, which is at room temperature, must be kept to a minimum. All these factors make it difficult to figure out what happens in large-scale SFQ circuits.

We diagnosed circuits using shift registers modified to enable us to identify error locations from output waveforms, which helps reveal the origins of circuit errors. The goal of our circuit diagnostics is to determine why the operating margins shrink in large-scale SFQ circuits.

\section{PRINCIPLE of CiRCUIT Diagnostics}

Shift registers are simple circuits that are often used as test circuits to investigate the effects of flux trapping [9]. However, output waveforms from conventional shift registers indicate only the existence of circuit errors but not their locations. Semenov et al. succeeded in identifying the properties of individual d-flip flop (DFF) cells by applying an independent bias to DFF cells [10]. We propose a simpler method of diagnosing circuits. Fig. 1(a) is a block diagram of our circuit. The output pulses from each DFF are merged into a serial pulse stream by confluence buffers (CBs) and monitored at output port "mout." We call this circuit the diagnostic shift register (DSR).

When a test sequence of " 1000 ..." is applied to the input port "din" of a 40-bit DSR, the correct operation produces a 40-bit data shift for the clock pulses at output "dout". At the same time, a sequence of "1111..." will appear at output "mout", where the total number of "1" must be 40 [Fig. 1(b)]. If a timing error occurs in the circuit, the number of data shifts at output "dout" will decrease, and the absence of an output pulse from the "1111..." sequence will be observed in output "mout" [Fig. 1(c)]. Here, the absence of an output pulse at a location indicates where the 


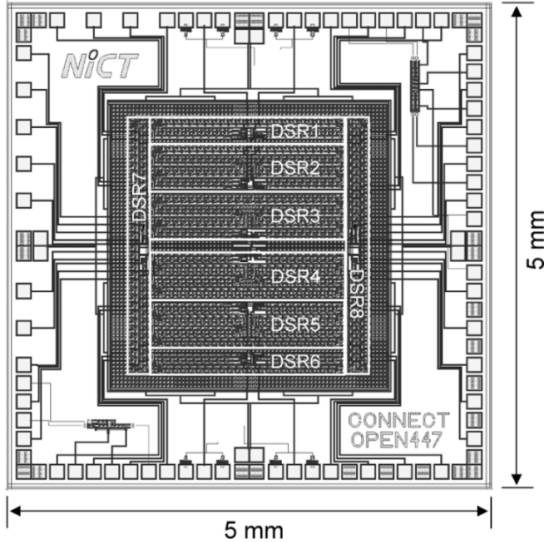

Fig. 2. Layout of test chip. Test circuit consists of eight diagnostics shift register blocks separated at locations other than bias lines.

(a)

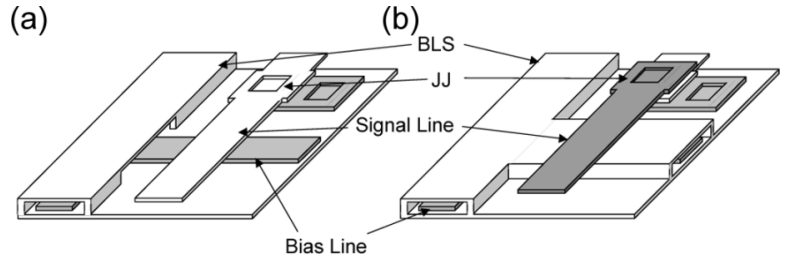

Fig. 3. Schematics of circuit layouts for (a) OPEN cells and (b) SUSHI cells.

timing error occurred. On the other hand, if the number of data shifts increases, it indicates that a clock pulse failed to trigger a DFF despite its internal state of " 1 ". When this happens, the output sequence of " $1111 \ldots$.." is divided at the error location. For example, Fig. 1(d) tells us that a sequence of three clock pulses failed to trigger the DFF at the 29th bit. Thus, we can determine error locations and their origins by observing the waveforms at outputs "mout" and "dout". Note that output "mout" is only used to check error locations and the bias margin in this paper is defined by the waveform from output "dout."

\section{EXPERIMENTAL RESULTS}

We designed and tested a large-scale DSR with more than 10,000 JJs. Fig. 2 shows the chip layout. The chip was fabricated using an NEC $2.5 \mathrm{kA} / \mathrm{cm}^{2} \mathrm{Nb}$ standard process [11]. The test circuit consists of eight DSR blocks, each of which is separated other than the bias lines. There are shifts of 40 bits for DSR1 and DSR6, 55 bits for DSR7 and DSR8, and 80 bits for DSR2 $\sim 5$. We designed the circuit using the CONNECT SFQ cell library [12]. The schematics of (a) our previous cell and (b) our latest cell are shown in Fig. 3. Bias line shields (BLS) have been used since 2002 because they are effective in reducing magnetic couplings between bias lines and circuits [5]. In our previous cells, the BLSs could not be inserted in places where bias lines intersect with signal lines because there were only three superconducting layers in the previous NEC standard process, which was insufficient to accommodate the full BLS shown in Fig. 3(b). However, the superconducting layer added in 2003 made the full BLS possible. We call our previous and latest cells "OPEN" and "SUSHI (Superconducting Shield) " cells, respectively. We measured two types of large-scale DSRs, one consisting of OPEN cells and the other of SUSHI cells. There are 11,192 and 10,772 JJs in the OPEN and the SUSHI versions,
TABLE I

Bias Margins ObSeRved IN ShIFt Registers With OPEN Cells

\begin{tabular}{|c|c|c|c|}
\hline \multirow{2}{*}{ Circuit } & \multicolumn{3}{|c|}{ Bias margin (Center of operating range) } \\
\cline { 2 - 4 } & CHIP No1 D6 & CHIP No2 D6 & CHIP No3 D6 \\
\hline DSR1 & Not operational & $\pm 3.8 \%(1.16 \mathrm{~A})$ & $\pm 1.4 \%(1.15 \mathrm{~A})$ \\
\hline DSR2 & Layout miss & Layout miss & Layout miss \\
\hline DSR3 & Not operational & $\pm 23.2 \%(1.16 \mathrm{~A})$ & $\pm 24.7 \%(1.13 \mathrm{~A})$ \\
\hline DSR4 & $\pm 22.2 \%(1.16 \mathrm{~A})$ & $\pm 23.8 \%(1.16 \mathrm{~A})$ & $\pm 21.8 \%(1.12 \mathrm{~A})$ \\
\hline DSR5 & $\pm 7.5 \%(1.13 \mathrm{~A})$ & $\pm 9.9 \%(1.14 \mathrm{~A})$ & $\pm 7.5 \%(1.09 \mathrm{~A})$ \\
\hline DSR6 & Not operational & Not operational & Not operational \\
\hline DSR7 & Not operational & Not operational & Not operational \\
\hline DSR8 & Not operational & Not operational & Not operational \\
\hline
\end{tabular}

respectively. The slight difference in the number of JJs is due to a minor change in the circuit layout, which did not significantly affect calculated results of the current distributions in the bias line networks.

The bias current was evenly supplied to the circuit from four sides. On each side, return currents in the ground plane were extracted from the neighboring ground pad to eliminate their effects on circuit operation [5], [7]. The optimal bias currents were $1.27 \mathrm{~A}$ and $1.23 \mathrm{~A}$ for the circuits with OPEN and SUSHI cells, respectively. Based on our previous results, stray magnetic fields due to off-chip currents around bias pads are estimated to be less than $10 \mu \mathrm{T}$ in the chip layout shown in Fig. 2, where the circuit is $0.8 \mathrm{~mm}$ from the bias pads and the maximum bias current is as large as $400 \mathrm{~mA}$ on each side [13]. We have also found that a magnetic field of $10 \mu \mathrm{T}$ does not significantly degrade circuit operation. Therefore, the effect of stray magnetic fields will be negligible in these circuit diagnostics.

We took all measurements using a cryoprobe with sixty non-magnetic pins made of $\mathrm{BeCu}$. The cryoprobe was equipped with a single-layer cylindrical permalloy metal shield that was $30.0 \mathrm{~cm}$ tall, $5.0 \mathrm{~cm}$ in diameter, and had a $0.01 \mathrm{~cm}$-thick wall. We inserted the cryoprobe directly into a He container (CRYOFAB, Inc. CMSH 60) and measured test chips.

\section{A. Shift Registers With OPEN Cells}

We measured three chips for circuits with OPEN cells. The results are summarized in Table I. The DSR3 and DSR4, which are located around the center of the circuit, come close to reproducing the bias margins of $+/-20 \sim 25 \%$. The DSR3 on chip No1 D6 probably includes a defect, because deflux did not improve circuit operation at all. Here, deflux is the procedure of eliminating trapped magnetic flux by warming the chip above the superconducting transition temperature $\left(T_{\mathrm{C}}\right)$ and slowly cooling it to $4.2 \mathrm{~K}$. In contrast, bias margins are $+/-7 \sim$ $10 \%$ for DSR 5 and less than $+/-5 \%$ for DSR 1 , indicating that the circuits closer to the periphery have lower bias margins. For DSR6, DSR7, and DSR8, we found no operational circuits in the test chips we measured. These results were reproduced several times after deflux. This reproducibility between chips and measurements suggests that the reduction or disappearance of the bias margin does not arise from defects or flux trapping.

Fig. 4 shows typical output waveforms for the bias currents outside the operating ranges. The circuits malfunction at around the 20th bit for DSR1 and the 40th bit for DSR5. These behaviors are reproducible for the other two chips we measured. These error locations agree well with the locations where calculation of the current distributions in the circuits predicted a 
(a)
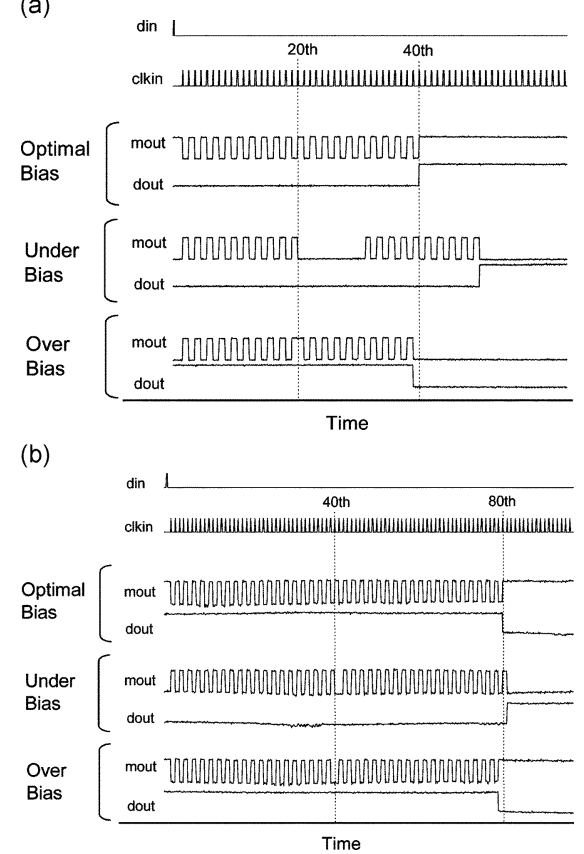

Fig. 4. Output waveforms in (a) DSR1 and (b) DSR5.

large amount of current would flow. The calculated maximum currents were approximately $6.5 \mathrm{~mA}$ in DSR1 (DSR6), $4.5 \mathrm{~mA}$ in DSR2 (DSR5), and $2.5 \mathrm{~mA}$ in DSR3 (DSR4). To confirm whether bias line currents of these orders really affect the circuit operations, we measured bias margins of the DFF cell while applying an additional current independent of the bias current to its bias line.

We chose 8-bit SRs as the test circuits. The detailed procedure of this measurement is described in a previous paper [5]. Each DFF cell has one vertical and two horizontal bias lines, where the BLS is applied to the vertical bias line in SUSHI cells but not in OPEN cells. Fig. 5(a) and (b) indicate the measured bias margins for the OPEN and SUSHI cells, respectively, while applying an additional current to the vertical bias line $\left(I_{\mathrm{BL}}\right)$. "Upward" and "downward" in Fig. 5 represent the $I_{\mathrm{BL}}$ direction. As the 8-bit SRs have eight vertical bias lines, we calculated current distribution to evaluate the current on each bias line. $I_{\mathrm{BL}}$ in Fig. 5 represents the maximum in the calculated bias line currents. The bias margins for an $I_{\mathrm{BL}}$ of less than $1 \mathrm{~mA}$ are around $+/-33 \%$ for both OPEN and SUSHI cells. The bias margins for OPEN cells are rapidly reduced by $I_{\mathrm{BL}} \mathrm{S}$ as large as $5 \mathrm{~mA}$, while no significant margin reduction is observed in SUSHI cells. The bias margins for OPEN cells are reduced to approximately $+/-20 \%$ for $I_{\mathrm{BL}}=2.5 \mathrm{~mA},+/-5 \%$ for $I_{\mathrm{BL}}=4.5 \mathrm{~mA}$, and almost zero for $I_{\mathrm{BL}}=5.5 \mathrm{~mA}$, which are in good agreement with the relationship between the observed bias margins in the large-scale DSRs and the calculated maximum currents described in the previous paragraph. These results support the conclusion that the observed reductions in bias margins in the large-scale DSRs with OPEN cells are caused by the magnetic fields from the bias line currents. We also applied an additional current to two horizontal bias lines but observed no considerable reduction of the bias margins in either OPEN cells or SUSHI cells. (a)

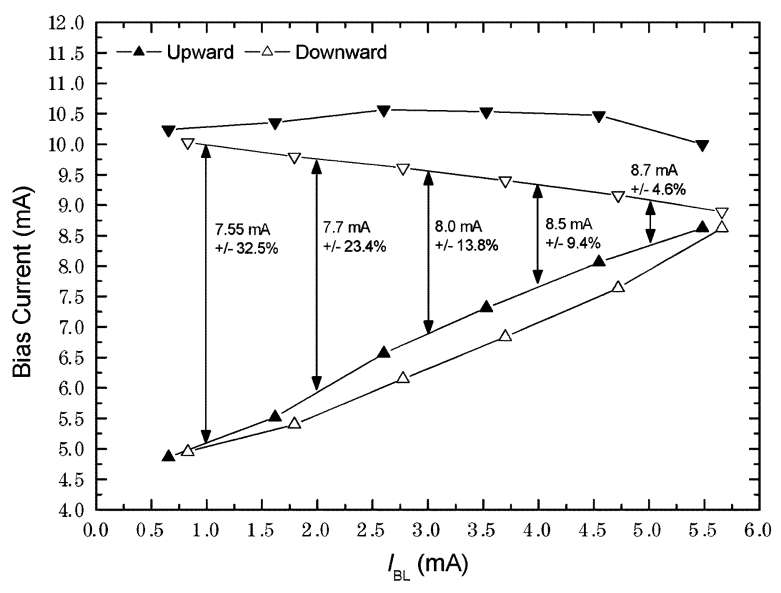

(b)

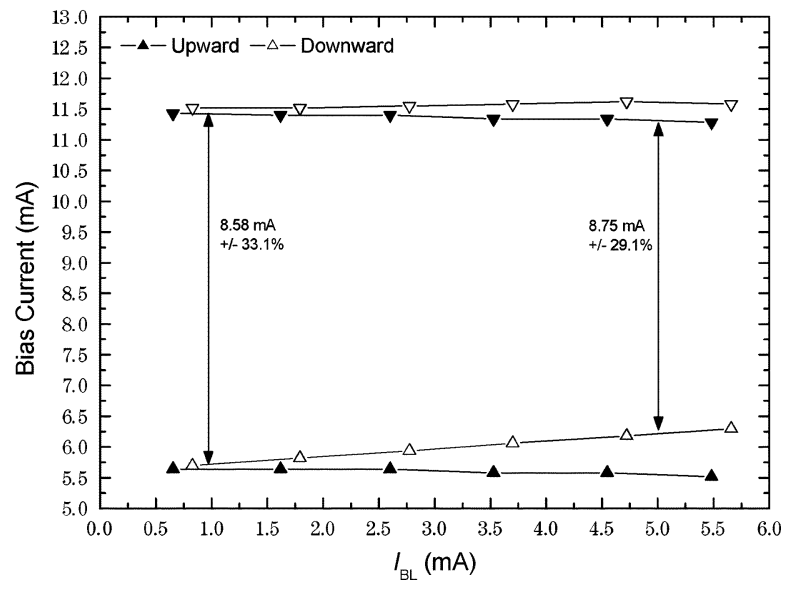

Fig. 5. Operating bias ranges of 8-bit shift registers as a function of $I_{\mathrm{BL}}$ for circuits made of (a) OPEN cells and (b) SUSHI cells.

TABLE II

Bias MARgINS ObSERVED IN SHIFT REgISTERS WITH SUSHI CELLS

\begin{tabular}{|c|c|c|c|}
\hline \multirow{2}{*}{ Circuit } & \multicolumn{4}{|c|}{ Bias margin (Center of operating range) } \\
\cline { 2 - 4 } & CHIP No2 D6 & CHIP No3 D6 & CHIP No4 D6 \\
\hline \multicolumn{4}{|c|}{ CryoFab 60L He Container with single-layer magnetic shield } \\
\hline DSR1 & $\pm 30.4 \%(1.06 \mathrm{~A})$ & $\pm 29.4 \%(1.08 \mathrm{~A})$ & $\pm 30.6 \%(1.14 \mathrm{~A})$ \\
\hline DSR2 & $\pm 1.6 \%(0.93 \mathrm{~A})$ & $\pm 25.6 \%(1.06 \mathrm{~A})$ & $\pm 32.0 \%(1.17 \mathrm{~A})$ \\
\hline DSR5 & $\pm 32.5 \%(1.05 \mathrm{~A})$ & $\pm 7.4 \%(0.92 \mathrm{~A})$ & $\pm 33.2 \%(1.14 \mathrm{~A})$ \\
\hline DSR6 & $\pm 31.2 \%(1.06 \mathrm{~A})$ & $\pm 16.1 \%(0.92 \mathrm{~A})$ & $\pm 31.6 \%(1.15 \mathrm{~A})$ \\
\hline \multicolumn{4}{|c|}{ FRP He dewar with dual-layer magnetic shield } \\
\hline DSR1 & $\pm 31.2 \%(1.06 \mathrm{~A})$ & $\pm 31.7 \%(1.11 \mathrm{~A})$ & $\pm 27.7 \%(1.11 \mathrm{~A})$ \\
\hline DSR2 & $\pm 1.1 \%(0.93 \mathrm{~A})$ & $\pm 31.2 \%(1.14 \mathrm{~A})$ & $\pm 32.1 \%(1.18 \mathrm{~A})$ \\
\hline DSR5 & $\pm 32.9 \%(1.06 \mathrm{~A})$ & $\pm 31.4 \%(1.08 \mathrm{~A})$ & $\pm 28.7 \%(1.07 \mathrm{~A})$ \\
\hline DSR6 & $\pm 31.9 \%(1.06 \mathrm{~A})$ & $\pm 26.9 \%(1.05 \mathrm{~A})$ & $\pm 31.6 \%(1.16 \mathrm{~A})$ \\
\hline
\end{tabular}

\section{B. Shift Registers With SUSHI Cells}

We measured three chips with test circuits made of SUSHI cells. The results are summarized in Table II. Because of layout misses, only the DSRs indicated in Table II (DSR1, 2, 5, and 6) were measured. All of the DSRs on chip No 4 D6 had bias margins of more than $+/-30 \%$, while the DSR1 and DSR6 are located around the periphery, indicating that using SUSHI cells significantly improves tolerance for bias line currents, as predicted from the results shown in Fig. 5(b). However, several DSRs on the other two chips had bias margins of less than $+/-20 \%$. Bias margins for chips No 2 D6 and No 3 D6 differed every time we measured them after deflux. In addition, the 
error locations identified from output waveforms also differed for every measurement, implying that flux trapping degrades the bias margins.

To reduce the effect of flux trapping, we measured the same chips in a fiber-reinforced-plastic (FRP) dewar surrounded by a dual-layer permalloy metal shield. The cooling speed of the test chip was also controlled using an automatic heat elevation system to identically reproduce the cooling in every measurement. The cooling speed was set to take about 15 minutes to decrease from just above $T_{\mathrm{C}}$ to $4.2 \mathrm{~K}$. For chip No 3 D6, bias margins seemed to improve, but slight effects of flux trapping were observed in DSR6. Bias margins are almost reproduced for chip No 2 D6, and a bias margin of a few percent was also reproduced in DSR2, suggesting that DSR2 in chip No 2 D6 was defective. For chip No 4 D6, slight effects of flux trapping were observed in DSR1 and DSR5, but were not observed in the measurement using the CMSH60 He container. We also examined slow cooling using the automatic elevating system in the measurement using the CMSH60 He container, but observed no remarkable differences. We did not try the FRP dewar for the circuits with OPEN cells. However, no significant change will ever be observed, because several measurements after deflux, even those using the CMSH60 He container, came close to reproducing the bias margins shown in Table I.

In this experiment, we paid little attention to size, shape, and distance between ground plane moats, even though it is well known that flux trapping dramatically depends on them [9], [14]. In addition, although a commercial gauss meter estimates the magnetic field inside the magnetic shield to be less than $1 \mathrm{mG}$, more precise measurement and reduction of the residual magnetic field will be necessary. Thermally induced currents also cause flux trapping, but their effect is not well understood. All these factors are important issues for the next stage of research.

\section{Fabrication Yield}

We now comment briefly on fabrication yields. Through our circuit diagnostics, we have tested a total of 24 DSRs on six chips. DSR2 and DSR $6 \sim 8$, which are made of our OPEN cells, are not counted because they failed to operate for reasons irrelevant to fabrication yields. Among the 24 tested DSRs, we found two DSRs that did not operate due to fabrication defects. If each circuit has one defect, which is reasonable considering that the other 22 circuits had no defect, there are two defects in the 24 DSRs we measured. There is a total of 34,698 JJs in the 24 DSRs. Therefore, the probability of finding a defect in a circuit with $10,000 \mathrm{JJs}$ is estimated to be $57.6 \%$.

\section{CONCLUSION}

We designed and tested shift registers for circuit diagnostics to improve operating margins of large-scale SFQ circuits. We fabricated two types of shift registers, one made with our previous cells and one with SUSHI cells. The circuits were diagnosed while investigating the reproducibility of chips and taking measurements after deflux. The output waveforms enable tenta- tive identification of the locations of circuit errors, which helped reveal where the errors originated. The test results indicated that the operations of the test circuits made of our previous cells are affected by leakage of magnetic fields from the bias lines, especially around the periphery where more currents flow in the bias lines. In contrast, no reductions of the bias margins were observed in any part of the circuit area in the circuits made of the SUSHI cells. However, flux trapping often degraded the bias margins even when we used a non-magnetic FRP dewar surrounded by a dual-layered permalloy metal shield. We found a total of two fabrication defects in the circuits with 34,698 JJs, indicating that the probability of finding a defect in a circuit with 10,000 Josephson junctions is less than $60 \%$.

\section{ACKNOWLEDGMENT}

The authors thank all CONNECT staff for their fruitful discussions.

\section{REFERENCES}

[1] O. A. Mukhanov, D. Gupta, A. M. Kadin, and V. K. Semenov, "Superconductor analog-to-digital converters," IEEE Trans. Appl. Supercond., vol. 92, pp. 1564-1584, 2004.

[2] M. Tanaka, T. Kawamoto, Y. Yamanashi, Y. Kamiya, A. Akimoto, K. Fujiwara, A. Fujimaki, N. Yoshikawa, H. Terai, and S. Yorozu, "Design of a pipelined 8-bit-serial single-flux-quantum microprocessor with multiple ALUs," Supercond. Sci. Technol., vol. 19, pp. 344-349, 2006.

[3] H. Terai, S. Yorozu, A. Fujimaki, N. Yoshikawa, and Z. Wang, "A new design approach based on a multi-wiring-layer process for high-density SFQ circuits," Supercond. Sci. Technol., vol. 16, pp. 1-6, 2003.

[4] M. Hidaka, S. Nagasawa, T. Satoh, K. Hinode, and Y. Kitagawa, "Current status and future prospect of the Nb-based fabrication process for single flux quantum circuits," Supercond. Sci. Technol., vol. 19, pp. $138-142,2006$.

[5] H. Terai, Y. Kameda, S. Yorozu, A. Fujimaki, and Z. Wang, "The effects of dc bias current in large-scale SFQ circuits," IEEE Trans. Appl. Supercond., vol. 13, pp. 502-506, 2003.

[6] M. Suzuki, M. Maezawa, and F. Hirayama, "Effects of magnetic fields induced by bias currents on operation of RSFQ circuits," Physica $C$, vol. 412-414, pp. 1576-1579, 2004.

[7] A. M. Kadin, R. J. Webber, and S. Sarwana, "Effects of superconducting return currents on RSFQ circuit performance," IEEE Trans. Appl. Supercond., vol. 15, pp. 280-283, 2005.

[8] E. Tolkacheva, H. Engseth, I. Kataeva, and A. Kidiyarova-Shevchenko, "Influence of the bias supply lines on the performance of RSFQ circuits," IEEE Trans. Appl. Supercond., vol. 15, pp. 276-279, 2005.

[9] R. P. Robertazzi, I. Shiddiqi, and O. Mukhanov, "Flux trapping experiments in single flux quantum shift registers," IEEE Trans. Appl. Supercond., vol. 7, pp. 3164-3167, 1997.

[10] V. K. Semenov, Y. A. Polyakov, and W. Chao, "Extraction of impacts of fabrication spread and thermal noise on operation of superconducting digital circuits," IEEE Trans. Appl. Supercond., vol. 9, pp. 4030-4033, June 1999.

[11] S. Nagasawa, Y. Hashimoto, H. Numata, and S. Tahara, "A 380 ps, 9.5 mW Josephson 4-Kbit RAM operated at a high bit yield," IEEE Trans. Appl. Supercond., vol. 5, pp. 2447-2450, June 1995.

[12] S. Yorozu, Y. Kameda, S. Tahara, H. Terai, A. Fujimaki, and T. Yamada, "A single flux quantum standard logic cell library," Physica $C$, vol. 378-381, pp. 1471-1474, Oct. 2002.

[13] H. Terai, S. Yorozu, A. Fujimaki, N. Yoshikawa, and Z. Wang, "Signal integrity in large-scale single-flux-quantum Circuit," Physica C, vol. 445-448, pp. 1003-1007, 2006.

[14] M. Jeffery, T. Van Duzer, J. R. Kirtley, and M. B. Ketchen, "Magnetic imaging of moat-guarded superconducting electronic circuits," Appl. Phys. Lett., vol. 67, pp. 1769-1771, 1995. 